\title{
Video Article \\ Purification and Analytics of a Monoclonal Antibody from Chinese Hamster Ovary Cells Using an Automated Microbioreactor System
}

\author{
Sai Rashmika Velugula-Yellela ${ }^{1}$, David N. Powers ${ }^{1}$, Phillip Angart ${ }^{1}$, Anneliese Faustino ${ }^{1}$, Talia Faison ${ }^{1}$, Casey Kohnhorst ${ }^{1}$, Erica J. Fratz-Berilla ${ }^{1}$, \\ Cyrus D. Agarabi ${ }^{1}$ \\ ${ }^{1}$ Center for Drug Evaluation and Research, Office of Product Quality, Office of Biotechnology Products, Division of Biotechnology Review and Research II, U.S. Food \\ and Drug Administration
}

Correspondence to: Cyrus D. Agarabi at Cyrus.Agarabi@fda.hhs.gov

URL: https://www.jove.com/video/58947

DOI: doi:10.3791/58947

Keywords: micro-bioreactor, Chinese hamster ovary cells, cell culture, screening, glycans, monoclonal antibody, size exclusion chromatography, multi-angle light scattering

Date Published: 12/7/2018

Citation: Velugula-Yellela, S.R., Powers, D.N., Angart, P., Faustino, A., Faison, T., Kohnhorst, C., Fratz-Berilla, E.J., Agarabi, C.D. Purification and Analytics of a Monoclonal Antibody from Chinese Hamster Ovary Cells Using an Automated Microbioreactor System. J. Vis. Exp. (), e58947, doi:10.3791/58947 (2018).

\section{Abstract}

Monoclonal antibodies (mAbs) are one of the most popular and well-characterized biological products manufactured today. Most commonly produced using Chinese hamster ovary $(\mathrm{CHO})$ cells, culture and process conditions must be optimized to maximize antibody titers and achieve target quality profiles. Typically, this optimization uses automated microscale bioreactors $(15 \mathrm{~mL})$ to screen multiple process conditions in parallel. Optimization criteria include culture performance and the critical quality attributes (CQAs) of the monoclonal antibody (mAb) product, which may impact its efficacy and safety. Culture performance metrics include cell growth and nutrient consumption, while the CQAs include the mAb's N-glycosylation and aggregation profiles, charge variants, and molecular weight. This detailed protocol describes how to purify and subsequently analyze HCCF samples produced by an automated microbioreactor system to gain valuable performance metrics and outputs. First, an automated protein A fast protein liquid chromatography (FPLC) method is used to purify the mAb from harvested cell culture samples. Once concentrated, the glycan profiles are analyzed by mass spectrometry using a specific platform (refer to the Table of Materials). Antibody molecular weights and aggregation profiles are determined using size exclusion chromatography-multiple angle light scattering (SEC-MALS), while charge variants are analyzed using microchip capillary zone electrophoresis (mCZE). In addition to the culture performance metrics captured during the bioreactor process (i.e., culture viability, cell counts, and common metabolites including glutamine, glucose, lactate, and ammonia), spent media is analyzed to identify limiting nutrients to improve the feeding strategies and overall process design. Therefore, a detailed protocol for the absolute quantification of amino acids by liquid chromatography-mass spectrometry (LC-MS) of spent media is also described. The methods used in this protocol take advantage of high-throughput platforms that are compatible for large numbers of small-volume samples.

\section{Introduction}

Protein therapeutics are being used to treat a growing variety of medical conditions including tissue transplant complications, autoimmune disorders, and cancers ${ }^{1}$. Since 2004, the United States Food and Drug Administration (USFDA) has documented an increasing proportion of biologic license applications (BLAs) of all approvals regulated by the Center for Drug Evaluation and Research (CDER), with BLAs accounting for over $25 \%$ in 2014 and $2015^{2}$

Considering this expanding market, biopharmaceutical manufacturers are challenged with quickly delivering more product with consistent quality. Efforts to augment product yield have focused on $\mathrm{CHO}$ cell engineering and production line screening, though the most significant improvements are due to advances in media/feed strategy optimization and cell culture environmental controls ${ }^{1,3,4,5}$ during the manufacturing process.

Since mAbs are produced in a biological system, there can be inherent protein variability. Antibody composition can be altered posttranslationally, such as glycosylation or impacted by degradation or enzymatic reactions. These structural variations may provoke dangerous immune reactions or alter antibody binding, which in turn can reduce or eliminate the intended therapeutic function ${ }^{5}$. Thus, critical quality attributes (CQAs) of monoclonal antibodies - N-glycan profile, charge variant distribution, and the percentage of antibody in monomeric form - are regularly monitored and controlled as part of a Quality by Design (QbD) approach during manufacturing processes ${ }^{1,6}$. In a regulated production environment, therapeutic proteins must meet acceptance criteria to be licensed as an approved commercial drug product ${ }^{7}$. The methods presented herein would typically be part of the quality characterization process for an antibody ${ }^{7,8}$, and any protein scientist will be familiar with their usage.

In prior work ${ }^{9}$, the application and operation of microbioreactors for high throughput screening of cell culture conditions in upstream bioprocessing has been described. The purified product obtained from the varying media conditions is subjected to $\mathrm{N}$-glycan analysis using LC-MS. Glycosylation patterns of therapeutic proteins can be detected and characterized using LC-MS techniques ${ }^{10,11}$, and the presence of various glycan species has been linked to bioprocess parameters such as feed strategy, $\mathrm{pH}$, and temperature ${ }^{12}$. The effect of the varying media conditions on product quality, indicated by the percentage of the resulting IgG in monomeric form, is also evaluated with Size Exclusion Chromatography- Multi-Angle Light Scattering (SEC-MALS) ${ }^{13,14,15}$. The charge variant profile represents a number of modifications ${ }^{16}$ that 
could impact the function of a product. Microcapillary zone electrophoresis (mCZE) is a technique that offers a considerably faster analysis time compared to traditional cation exchange (CEX) chromatography and capillary isoelectric focusing (cIEF) methods used for charge variant analysis $^{17,18}$. Spent bioreactor media was analyzed to track amino acid consumption during protein production as it relates to changes in the antibody's identifying attributes ${ }^{19,20,21,22,23}$

Protein analytics allow us to identify critical process parameters (CPPs) based on the relationships between process inputs and changes in CQAs. During bioprocess development, identifying and measuring CPPs fundamentally demonstrates process control and ensures that the product has not changed, which is essential in highly regulated manufacturing environments. In this paper, analytical techniques to measure some of the biochemical characteristics of the protein most pertinent to product CQAs ( $\mathrm{N}$-glycan profile, charge variants, and size homogeneity) are presented.

\section{Purification of antibody}

NOTE: The equilibration buffer for the in-house antibody is $25 \mathrm{mM}$ Tris, $100 \mathrm{mM} \mathrm{NaCl}, \mathrm{pH}$ 7.5. The elution buffer used is $0.1 \mathrm{M}$ acetic acid. The buffers and resin (Protein A) are dependent on the specific antibody purified. Column volume is equivalent to the bed height of the resin. The amount of mobile phase used is determined in terms of column volume.

1. Initializing the purification system

1. Open the software attached to the purification system. Using manual instructions, equilibrate the column with the equilibration buffer at a flow rate of $2 \mathrm{~mL} / \mathrm{min}$ for $40 \mathrm{~min}$. Stop the manual run after equilibration.

2. In the fraction collector, place $15 \mathrm{~mL}$ conical tubes to collect purified antibody eluate and $50 \mathrm{~mL}$ conical tubes to collect flow-through during high salt wash. Ensure that the fraction collector is reset to the start position by opening and closing the fraction collector before the beginning the run. The fraction collector is maintained at $7^{\circ} \mathrm{C}$.

NOTE: The fraction collector can be reset manually under the Fraction Collector tab in Settings, for both $15 \mathrm{~mL}$ and $50 \mathrm{~mL}$ tubes.

2. Sample injection

NOTE: The harvested cell culture fluid used in the following procedures has been obtained from chinese hamster ovary cells cultured in automated micro-bioreactors ${ }^{9}$.

1. Add the $0.22 \mu \mathrm{m}$ filtered harvest cell culture fluid to an empty $12 \mathrm{~mL}$ syringe whose nozzle end is capped.

2. Holding the syringe with the nozzle facing down, insert the syringe plunger till a small portion of the plunger is in. Making sure that the fluid is not leaking, turn the syringe with the nozzle facing up and remove the cap.

3. Still holding the syringe with the nozzle facing up, push the cylinder to dispel any air until the cell culture fluid is at the tip of the nozzle. Insert the syringe nozzle into the manual injection port on the purification system and twist to tighten.

4. Push down on the plunger until all the sample is injected and is visible in the attached $10 \mathrm{~mL}$ large volume sample loop.

5. Open the saved method file. Save the result file in the required location and specify file name when prompted. Hit run after the sample has been injected into the large volume sample loop.

3. Running the purification method

1. Select the saved method and click run when prompted by instrument software (step 1.2.5).

NOTE: The system is set up to run the following steps. The user needs not do anything while the instrument is running.

2. Equilibrate the column with three column volumes $(\mathrm{CVs})$ of equilibration buffer at a flow rate of $2 \mathrm{~mL} / \mathrm{min}$. Once the column is equilibrated, the system, using the large volume sample loop, will inject the sample onto the column at a flow rate of $1 \mathrm{~mL} / \mathrm{min}$.

3. A drop in UV signal at $280 \mathrm{~nm}$ indicates that the sample is finished loading. Wash the column with equilibration buffer at a flow rate of 2 $\mathrm{mL} / \mathrm{min}$ until the UV signal drops below $25 \mathrm{mAU}$.

4. Use four $\mathrm{CVs}$ of $25 \mathrm{mM}$ Tris with $1 \mathrm{M} \mathrm{NaCl}$ at $\mathrm{pH} 7.5$ to perform a secondary high salt wash at a flow rate of $2 \mathrm{~mL} / \mathrm{min}$. The system fraction collector will collect any protein/DNA that comes off the column during the salt wash in $50 \mathrm{~mL}$ tubes.

5. Apply five CVs of elution buffer at a flow rate of $1 \mathrm{~mL} / \mathrm{min}$ to elute the antibody off the column. Collect the eluate in $15 \mathrm{~mL}$ tubes based on UV signal; when the UV 280 signal is above $35 \mathrm{mAU}$, collection starts; collection ends when the signal drops below $50 \mathrm{mAU}$; this is called peak cutting.

NOTE: Peak cutting ensures normalization of elution profiles and to avoid elution peak tailing which may contain protein aggregates ${ }^{24}$.

6. Wash the column using three CVs of equilibration buffer. The run ends after the wash step.

7. After elution immediately neutralize the purified protein using $1 \mathrm{M}$ Tris base to a $\mathrm{pH}$ of $\sim 5.5$. Measure the protein concentration using a microvolume UV-Vis spectrophotometer at $280 \mathrm{~nm}$ and $260 \mathrm{~nm}$ and store at $4{ }^{\circ} \mathrm{C}$.

8. Concentrate the purified antibody using centrifugal units (step 2). Then subject the purified antibody to glycan analysis using LC-MS and after aggregation profile analyze using SEC-MALS (steps $3 \& 4$ ).

NOTE: The purified antibody should not be frozen without further buffer exchange as frequent freeze-thaw cycles can cause aggregation and precipitation.

\section{Concentration of purified antibody}

NOTE: The Tris-acetate buffer is $0.1 \mathrm{M}$ acetic acid neutralized with $1 \mathrm{M}$ Tris Base to a $\mathrm{pH}$ of $\sim 5.5$.

1. Insert $100 \mathrm{kDa}$ filters into centrifuge tubes. Centrifuge for $10 \mathrm{~min}$ at $14,000 \mathrm{xg}$ at room temperature (RT).

2. Wash the filters with $500 \mu \mathrm{L}$ of double distilled water. Centrifuge for $10 \mathrm{~min}$ at $14,000 \times \mathrm{g}$. Repeat this step twice. Discard filtrate.

3. Transfer the rinsed filters to fresh centrifuge tubes and add $500 \mu \mathrm{L}$ of sample to each filter. Centrifuge for $10 \mathrm{~min}$ at $14,000 \times g$. 
4. Invert the filter into a fresh spin tube. Centrifuge for $2 \mathrm{~min}$ at $1,000 \times g$ to collect the concentrated sample.

5. Determine the sample concentrations using a UV-Vis spectrophotometer. Blank the spectrophotometer using a solution of Tris-acetate buffer. Use a protein extinction coefficient of $1.37 \mathrm{~mL}^{*}\left(\mathrm{mg}^{*} \mathrm{~cm}\right)^{-1}$ at $280 \mathrm{~nm}$ for a $1 \%(\% \mathrm{~m} / \mathrm{v}) \mathrm{lgG}$ solution.

6. Use the concentrated sample to prepare $12.5 \mu \mathrm{L}$ of $2 \mathrm{mg} / \mathrm{mL}$ working solution for glycan analysis and $30 \mu \mathrm{L}$ of $3.5 \mathrm{mg} / \mathrm{mL}$ working solution for SEC-MALS.

NOTE: The protocol can be paused here. Samples should be refrigerated at $4{ }^{\circ} \mathrm{C}$. At $2 \mathrm{mg} / \mathrm{mL}$ concentration, these samples should be stable for at least three months at $4^{\circ} \mathrm{C}$ while higher concentrations might precipitate.

\section{Analysis of $\mathrm{N}$-glycans using mass spectroscopy}

1. N-Glycan labeling and isolation

1. Start with antibody concentrations of $2 \mathrm{mg} / \mathrm{mL}$ in an appropriate buffer such as neutral sodium phosphate, citrate or HEPES buffer. Prepare an intact mAb standard (such as NIST mAb) at $2 \mathrm{mg} / \mathrm{mL}$ to process alongside the experimental samples to serve as a positive control.

NOTE: The antibodies should be in a final buffer containing no SDS and less than $0.1 \mathrm{mM}$ nucleophiles (such as Tris, DTT, glycine or histidine). SDS in the sample buffer must be removed. If nucleophiles are in the buffer, then dilute them down or perform a buffer exchange since they will interfere with the kit. The general protocol is provided with the glycan kit.

2. Dilute $7.5 \mu \mathrm{L}$ of the antibodies with $15.3 \mu \mathrm{L}$ of LC-MS-grade water in $1 \mathrm{~mL}$ tubes provided with the kit and then denature using $6 \mu \mathrm{L}$ of $5 \%$ solution of an enzyme-friendly and MS-friendly surfactant at $90{ }^{\circ} \mathrm{C}$ for $3 \mathrm{~min}$.

3. Cool the samples for $3 \mathrm{~min}$ to room temperature (RT). Then, add $1.2 \mu \mathrm{L}$ of PNGase $\mathrm{F}$ and incubate for $5 \mathrm{~min}$ at $50{ }^{\circ} \mathrm{C}$.

4. After cooling $3 \mathrm{~min}$ to RT, label the cleaved $\mathrm{N}$-glycans by adding $12 \mu \mathrm{L}$ of fluorescent tagging reagent dissolved in anhydrous dimethylformamide (DMF) and wait for 5 min. Dilute the labeled N-glycan mixture with $358 \mu \mathrm{L}$ of acetonitrile (ACN).

5. Place a hydrophilic interaction chromatography (HILIC) plate in a vacuum manifold with shims and waste tray. Use a multichannel pipette for large numbers of samples.

6. Condition the wells with $200 \mu \mathrm{L}$ of water, where the vacuum is adjusted so the liquid will take $15-30 \mathrm{~s}$ to pass through the HILIC resin Equilibrate with $200 \mu \mathrm{L}$ of $85 \%$ ACN prior to loading the ACN-diluted labeled glycan mixture $(400 \mu \mathrm{L})$, applying the vacuum after each new liquid is added to the wells. Wash the resin with $600 \mu \mathrm{L}$ of $1 \%$ formic acid (FA) $/ 90 \%$ ACN twice.

7. Replace the waste tray with $600 \mu \mathrm{L}$ collection tubes. Elute the labeled N-glycans with SPE elution buffer ( 3 elutions of $30 \mu \mathrm{L}$ each) into the collection tubes. Dilute the pooled elutions with $310 \mu \mathrm{L}$ of DMF/ACN sample diluent. Pipette the samples into auto sampler vials to be ready for fluorescence (FLR)-MS analysis.

NOTE: These samples are stable when stored at $-80{ }^{\circ} \mathrm{C}$ for at least 1 month. Store the HILIC plate in its original packaging, taped shut and inside a desiccator for future use.

\section{LC-MS analysis of labeled N-glycans}

1. Analyze the labeled N-glycan elution samples on an Ultra Performance Liquid Chromatography (UPLC) system coupled to a fluorescence detector and quadrupole time-of-flight (Q-ToF) mass spectrometer. Use a column approved for chromatographic separation of the labeled glycans and heat to $60^{\circ} \mathrm{C}$ during separations.

NOTE: The column must be flushed with $60 \%$ acetonitrile and $40 \% \mathrm{H}_{2} \mathrm{O}$ before use: $50 \mathrm{CVs}$ before the first use or $20 \mathrm{CVs}$ if the column has been used before.

1. Use $50 \mathrm{mM}$ ammonium formate (AmF) (made with mobile phase concentrate) and $100 \%$ LC-MS-grade ACN for the mobile phases. The AmF is sensitive to $\mathrm{pH}$ changes and is usable for 1 month after mixing. Set the initial flow rate to $0.4 \mathrm{~mL} / \mathrm{min}$, with the LC gradient providing increasing AmF during the elution phase.

2. Set the FLR detector to measure at EX $265 / E M 425 \mathrm{~nm}$ with a sampling rate of $2 \mathrm{~Hz}$. Set the Q-ToF to MS1 positive ion sensitivity mode, with a mass range of $100-2,000$ daltons (Da), a scan time of 0.25 seconds and continuum data acquisition. Use leucine enkephalin ( $2 \mathrm{ng} / \mu \mathrm{L}$ in $50 \% \mathrm{ACN} / 0.1 \% \mathrm{FA}$ ) for the internal mass reference, in the "Do NOT apply correction" mode. NOTE: The internal mass reference correction will be applied later during data processing.

3. Re-suspend the dextran ladder sequentially in $22.5 \mu \mathrm{L}$ of $\mathrm{H}_{2} \mathrm{O}, 25 \mu \mathrm{L}$ of DMF and $52.5 \mu \mathrm{L}$ of ACN. Prepare $10 \mu \mathrm{L}$ aliquots for storage at $-80^{\circ} \mathrm{C}$, as the ladder is not stable for more than $24 \mathrm{~h}$ at higher temperatures (room temperature, $4{ }^{\circ} \mathrm{C}$ ). The dextran ladder degrades after more than one freeze-thaw cycle.

4. Place the samples in the auto sampler set to $10^{\circ} \mathrm{C}$. Load a vial of dextran ladder along with samples, as the retention time information of the ladder will be used for assignments while the mass information used to validate identifications. Use $10 \mu \mathrm{L}$ injections for the samples and $7.5 \mu \mathrm{L}$ injections for the ladder. Inject samples in triplicate. Run the loaded method.

\section{N-Glycan identification for LC-MS data}

1. Perform data processing with a program optimized for hydrophilic interaction chromatography fluorescence mass spectrometry (HILICFLR-MS) data.

2. Apply internal mass reference corrections within the program. Designate the dextran ladder injections as "Standard" in Sample Information. In the Analysis Method, set the Separation Compound retention times to those of the ladder compounds that were detected during the run.

3. To ensure that Area \% will be returned for identified glycans, modify the Analysis Method: Under the Processing tab, click Quantitation Settings - Calibrate and set "Calibration curve fit type" to "Relative response (\%)".

\section{Analysis of antibody aggregation using SEC-MALS}

1. Sample preparation

1. Transfer the $3.5 \mathrm{mg} / \mathrm{mL}$ (step 2) diluted protein to a vial with a $150 \mu \mathrm{L}$ glass insert. Use a gel loading tip to pipet into the bottom bell of the insert to avoid the introduction of bubbles. 
2. Cap the vial with a septa cap and analyze immediately. Store at $4{ }^{\circ} \mathrm{C}$ if analyzing later.

2. SEC-MALS configuration and equilibration

NOTE: Analyze aggregation on SEC-MALS configured with an Ultra High-Pressure Liquid Chromatography (UHPLC) with a MALS Detector and Refractive Index Detector controlled by the MALS software.

1. Configure a method file in the UHPLC software for control of the UHPLC system, setting the flow rate to $0.4 \mathrm{~mL} / \mathrm{min}$ with a $\mathrm{mobile}$ phase of $1 \mathrm{x}$ Phosphate Buffered Saline (PBS) (diluted from 10x), the injection volume to $5 \mu \mathrm{L}$, the column temperature to $25^{\circ} \mathrm{C}$, and the Diode Array Detector (DAD) to monitor $280 \mathrm{~nm}$. Set run time to $20 \mathrm{~min}$. Equilibrate the system for at least $4 \mathrm{~h}$ prior to any sample analysis.

2. The interface between UHPLC and Multi Angle Light Scattering - Refractive Index (MALS-RI) detectors requires the use of the analog output on the DAD. Set the DAD attenuation to $1000 \mathrm{mAU}$ in the DAD method file and AU/UV setting to 1 (UV instrument $>$ Channels $>$ Channel 1 ).

3. Turn on the DAD lamp 30 min prior to beginning analysis and set the wavelength to $280 \mathrm{~nm}$. At the same time, purge the Refractive Index (RI) reference cell for $15 \mathrm{~min}$ or until the baseline is stable and then close the reference cell.

4. Configure SEC-MALS software sequence, setting the collection time to $12 \mathrm{~min}$, the injection volume to $5 \mu \mathrm{L}$, the $\mathrm{dn} / \mathrm{dc}$ to $0.185 \mathrm{~mL} / \mathrm{g}$, A280 extinction coefficient if previously experimentally determined or to $1.37 \mathrm{~mL}^{*}\left(\mathrm{mg}^{*} \mathrm{~cm}\right)^{-1}$, and the concentration of the sample. Click Run and wait for the "waiting to inject" dialog to appear on the screen.

NOTE: The A280 extinction coefficient is specific to the protein of interest and should be determined experimentally.

5. Configure a sample list in the UHPLC software in the same order as in MALS-RI software and submit.

NOTE: It is important to run a system suitability check before and after a run. Bovine serum albumin typically is used to check for peak broadening, a sign that the SEC column may need cleaning or replacement. The same BSA standard injection can be used to specify signal alignment, peak broadening, and detector normalization.

\section{Aggregate analysis with MALS software}

1. Click on the tab marked Procedures. Specify the minimum level of despiking required; none is usually sufficient.

2. Verify that baselines have been drawn correctly and adjust if necessary, for LS1, LS2, LS3, RI, and UV channels. Set the peak area of interest.

3. Review the molecular mass distribution to confirm that the called peaks contain particles of similar size.

\section{Charge variant analysis}

1. Sample preparation and labeling

1. Start with $80 \mu \mathrm{L}$ of a $3.5 \mathrm{mg} / \mathrm{mL}$ antibody solution.Desalt the sample using a $0.5 \mathrm{~mL}$ Desalting Column (7k MWCO). Prepare the column by first snapping off the bottom stopper, then loosening the top stopper, and placing it in a $1.7 \mathrm{~mL}$ micro-centrifuge tube. Centrifuge the desalting column for $1 \mathrm{~min}$ at $1,500 \times \mathrm{g}$.

NOTE: Mark the exterior of the column with a dot so that it can be placed in the original orientation for the next steps.

2. Transfer the column to a new micro-centrifuge tube. Add the $80 \mu \mathrm{L}$ of diluted protein to the top of the column. Align the column to the original orientation. Centrifuge for $2 \mathrm{~min}$ at $1,500 \times \mathrm{g}$. Remove the sample from the centrifuge, discard the desalting column and mix the sample well.

NOTE: Desalting is only required if the sample matrix contains primary amines, excipients that will perturb the sample electrophoresis, or other incompatible substances.

3. Dilute the sample to a final concentration of $2 \mathrm{mg} / \mathrm{mL}$ in a volume of $25 \mu \mathrm{L}$ and add $5 \mu \mathrm{L}$ of the labeling buffer (see Table of Materials: Charge Variant Reagent Kit) in the 96-well plate. Prepare the labeling reagent by diluting the necessary amount of labeling reagent (see Table of Materials: Charge Variant Reagent Kit) 1:30 in dimethylformamide. Incubate the sample for 10 min at room temperature away from light.

NOTE: It is important to thaw and then immediately use this reagent and use it within 10 min of mixing with DMF.

4. After incubating, add $60 \mu \mathrm{L}$ of reagent grade water and mix well by pipetting. Cover the plate with a plate seal and centrifuge the plate at $1,000 \times g$ for $1 \mathrm{~min}$.

2. Preparing the charge variant chip

1. Prepare the Charge Variant chip by removing storage solution and washing wells $1,3,4,7$, 8 , and 10 with water. Then replace the water with $\mathrm{pH} 7.2$ running buffer (see Table of Materials: Charge Variant Reagent Kit).

2. Add $750 \mu \mathrm{L}$ of $\mathrm{pH} 7.2$ running buffer to the buffer tube and place the buffer tube in the indicated spot on the upper left-hand corner of the sample tray. Now remove the plate seal from the 96-well plate, press Unload Plate on the instrument user interface, and insert the plate into the GXII sample tray.

NOTE: $\mathrm{pH} 7.2$ buffers were used for this analysis. $\mathrm{pH}$ 5.6-7.2 buffers may be used depending on the protein pl. When using lower $\mathrm{pH}$ buffers, longer sample run times may be required.

3. Press the Unload Chip button on the user interface. Ensure that the electrodes are free of any particles, and if not, clean with a lint free swab. When inserting the chip make sure that the window in the center of the chip is free of particles or smudges. If necessary, clean with a lint-free soft cloth.

NOTE: When working with capillary electrophoresis chips, remove buffer by vacuum aspiration, followed by the immediate addition of the next solution to prevent wells from drying out. To minimize the introduction of bubbles, practice reverse pipetting technique. When handling the chip, be mindful of the fragile capillary extending from the bottom of the chip, making sure that it does not dry out and does not break through rough handling.

4. Close the lid to the chip chamber and select the HT Protein Charge Variant assay. Click the Run button. Follow the prompts to select the sample wells, plate type, assay time $(68,90$, or $100 \mathrm{~s})$, and file name. Click Start at the end of the prompts.

5. Chip cleanup requires the washing of each well $2 x$ with water, followed by the addition of Storage Buffer (see Table of Materials: Charge Variant Reagent Kit). Once in storage buffer, replace the chip in the instrument and, when prompted, select the HT Protein 
Charge assay. On the main screen select Wash on the user interface. Once completed, remove the chip, wipe down the electrodes with water and a lint-free swab, and store the chip at $4{ }^{\circ} \mathrm{C}$.

3. Charge variant analysis

1. Open the instrument analysis software. Import the run by going to File>Import Data File... and clicking on the desired *.gxd file. Only the name will be carried over to software, so renaming the wells is advantageous (Tools > Sample Name Editor). Select the files to be exported by holding down shift while selecting the files. Click File>Export... and select the Raw Data box and then the AlA Format box.

2. Open the Browse Projects tab within the analysis software. Click on Database>Import Data... and select the exported *.CDF files.

3. Once imported, navigate to the Injections tab, select the files to be analyzed, right click and go to Process... In the window that pops up, select the check box next to Process and select the "Use specified processing method" radio box and the desired processing method from the drop-down box. In the drop-down box immediately below labeled "How:" select Calibrate and Quantitate. Once processed, navigate to the Results tab and check the integration of the chromatograms.

NOTE: The processing method needs to be verified for each method. As a starting point, the parameters used for the current processing method are included in Supplementary file.

NOTE: Exporting of the data can be done in the form of a report or only the peak quantification may be exported. These can be done the same time as processing or from the results window.

\section{Amino acid analysis}

1. Setting up the standard curve for absolute amino acid quantification by LC-MS

1. Prepare the extended amino acid (EAA) mixture by dissolving $59.45 \mathrm{mg}$ of Asn, $59.00 \mathrm{mg}$ of Hyp, $65.77 \mathrm{mg}$ of Gln, and $91.95 \mathrm{mg}$ of Trp in $25 \mathrm{~mL}$ of $0.1 \mathrm{~N} \mathrm{HCl}$. The final concentration of each amino acid in the EAA mixture is $18 \mathrm{nmol} / \mu \mathrm{L}$.

2. Prepare the internal standard (ISTD) stock solution by dissolving $58.58 \mathrm{mg}$ of Nva and $44.54 \mathrm{mg}$ of Sar in $50 \mathrm{~mL}$ of $\mathrm{HCl}$.

3. Prepare the complete amino acid standards by combining the amino acid stock solution containing Ala, Asp, Arg, Cys, Glu, Gly, His, Ile, Leu, Met, Phe, Pro, Ser, Thr, Trp, Tyr, Val at $1 \mathrm{nmol} / \mu \mathrm{L}$ each with the EAA mixture for final amino acid concentrations of 900,225 , $90,22.5$, and $9 \mathrm{pmol} / \mu \mathrm{L}$. Add the prepared ISTD stock to the amino acid standards for a final concentration of either $90 \mathrm{pmol} / \mu \mathrm{L}$ or 900 $\mathrm{pmol} / \mu \mathrm{L}$ to create "low" and "high" internal standards to use as positive controls for the method.

4. Place the amino acid concentrations in sample vials into the autosampler of the UPLC. Generate a calibration curve $(9$ to $900 \mathrm{pmol} / \mu \mathrm{L})$ in the instrument software based on the amino acid standard concentrations using the following instructions.

5. Use the Q-ToF in electrospray ionization (ESI) positive sensitivity mode coupled to a UPLC for intact amino acid analysis. For chromatographic separation, use a normal phase column made for amino acid separations. Prepare the following buffers with mass spectrometry grade reagents: $A=$ acetonitrile $+0.1 \%$ formic acid and $B=100 \mathrm{mM}$ ammonium formate. Set the LC flow rate to $0.6 \mathrm{~mL} /$ min and column temperature to $40^{\circ} \mathrm{C}$.

6. Use the following 15-minute gradient conditions for amino acid separations: $14 \%$ B (0-3 min), $14-100 \%$ B (3-10 min), $100 \%$ B (10-13 $\min ), 100-8 \%$ B (13-14 $\min ), 8 \%$ B (14-15 min).

7. Use the "Sample Type" and "Conc A" column listings in the MS acquisition program to create a calibration curve for future analysis of crude bioreactor media in the quantitation program. To make these columns appear in acquisition program, use the Customize Display... command when right-clicking on the top menu bar.

8. The Sample Type for the amino acid standards will be "Standard" while the media samples will be "Analyte". Fill in the "Conc A" column with the numerical concentrations of the standards in the required units (ex: pmol/ $\mu \mathrm{L}$ ).

9. Run the prepared amino acid standard concentrations at least twice. Validate that the UPLC instrument and mass spectrometer are working properly by checking the ISTD peaks.

10. Use the "Edit Method" option in the quantitation application to create the quantitation method ( ${ }^{*}$.mdb file). Define all the amino acids of interest in the quantitation application, such as the compound name, $\mathrm{m} / \mathrm{z}$ value and expected retention time. Change the integration parameters for the method here.

11. Use the created amino acid method on the standard samples to create the calibration curve. This curve can be exported to a *.cdb file for use with the media samples using the Export>Calibration... command.

12. In the quantitation application, save the desired layout to a *.qlt file to apply to future datasets using "Save Layout As...". Name (Injection name), Area and Conc are the most important output columns.

2. Amino acid analysis of crude bioreactor media by LC-MS

1. Centrifuge crude bioreactor media at $1,962 \times g$ for 5 minutes and pass through a $0.22 \mu \mathrm{m}$ filter.

2. Follow up with a perchloric acid cleanup to remove protein and particulate matter: mix the filtered bioreactor media with $0.4 \mathrm{~N} \mathrm{HCIO}_{4}$ at a $1: 1$ ratio and centrifuge at $14,700 \times g$ for $5 \mathrm{~min}$ at $\mathrm{RT}$. Collect the clarified media in autosampler vials.

NOTE: Adjust the injection volume as needed to make the amino acid concentrations fall within the calibration range. Depending on the instrument, the injection volume can be adjusted between $0.1 \mu \mathrm{L}$ and $10 \mu \mathrm{L}$.

3. Run media samples in triplicate by LC-MS. Use "Process Samples" under the quantitation program along with the method (*.mdb) and calibration file ( $\left.{ }^{*} . \mathrm{cdb}\right)$. The method and calibration curve will be automatically applied to the crude media samples by the quantitation application once all the injections are completed.

4. To export data for analysis in another program (such as a spreadsheet), use the "Print" command and create an *.xps or *.pdf file. 


\section{Representative Results}

The harvested cell culture fluid from the automated microscale bioreactor is purified using fast protein liquid chromatography (FPLC), as seen in Figure 1 and the purified proteins' critical quality attributes (CQAs) were characterized by various downstream analytical methods. This is a key benefit of the automated microbioreactor system; differences in CQAs can be rapidly assessed across a wide range of conditions. N-glycan data from $\mathrm{CHO}$-produced mAbs that are processed by mass spectrometry should appear like the chromatograms shown in Figure 2. The figure depicts a comparison between two chromatograms showing that the mannose 5 peak (M5) from one sample is considerably lower. If only a noisy baseline is observed instead of peaks, this may mean that the chromatography setup is faulty or that the procedure is not successful. Using controls, troubleshooting can be simplified. First, assess the FLR peaks from the dextran ladder; these peaks indicate that the chromatographic system is working correctly. Next, compare the experimentally obtained peaks with those obtained from a processed intact mAb standard. If peaks from the standard are visible, but no sample peaks are identified, then the mAb samples were not processed correctly. This may be due to SDS or nucleophile presence in the buffer interfering with N-glycan labeling and purification.

SEC-MALS can be used to assess two more CQAs: the aggregation profile and the molecular weight of the antibody. A representative SECMALS chromatogram is comparable to the one shown in Figure 3. The molecular mass distribution and the absolute molecular weight were determined using the required software with an extinction coefficient of $1.37 \mathrm{~mL}^{*}\left(\mathrm{mg}^{*} \mathrm{~cm}\right)^{-1}$ and a dn/dc of $0.185 \mathrm{~mL} / \mathrm{g}$. As peak calling and setting the baseline in the software is performed manually, results may vary slightly from user to user. The absolute molecular weight of monomeric IgG1 from Figure 3 is $1.504 \times 10^{5} \mathrm{Da} \pm 0.38 \%$ (blue) and the higher order complex is $7.799 \times 10^{5} \mathrm{Da} \pm 3.0 \%$ (red). The polydispersity of the aggregates is much greater than that of the monomer, as indicated by the red molar mass distribution of Peak 1 (Figure 3). The small quantity of sample and importance of aggregation as a CQA make this technique a highly valuable complementary analytical tool to the automated microbioreactor system.

The result of $\mathrm{mCZE}$ is an electropherogram, such as in Figure 4, which shows the charge variant profile for a monoclonal antibody. The profile is a unique signature for the protein being investigated and is highly sensitive to the operating $\mathrm{pH}$. Also visible is a free-dye peak to the left of the charge variant profile. When establishing an operating $\mathrm{pH}$, there is some discretion to the operator to balance resolution and signal; in addition, the operator must ensure good separation from the free-dye peak which migrates at $\sim 30 \mathrm{~s}$. The sample can be desalted after labelling to remove this peak, though this leads to a significant loss in signal. Once an operating pH is established, the sample profiles can be compared. While generally consistent, changes in labeling efficiency or differences in excipients can lead to minor differences in the migration of a sample and the charge variant profile making electropherograms hard to directly compare. Instead, the method of comparison is usually based on the percentages of basic, main, and acidic species. In this case, relative differences as small as $1-2 \%$ can be identified using mCZE.

Amino acid consumption can be monitored to determine if depletion is causing changes in CQAs. Chromatogram readouts from the mass spectrometer can be used to evaluate the successful creation of a calibration curve for the absolute quantification of amino acids in crude bioreactor media samples. Figure 5 depicts two total ion chromatograms (TIC) and one extracted ion chromatogram (XIC) as representative results during this process. In Figure 5A, the TIC shown depicts the background signal from the buffer system as only a water blank was injected. Figure 5B depicts a representative TIC of the amino acid standard where, when compared to the water blank, small peaks that correspond to the individual amino acid species can be observed (such as lysine at 7.96 minutes). To integrate the peak and facilitate the quantification of peak area (and therefore the concentration), the XIC is used where only the signal from a defined "chromatogram mass window" is displayed. Depending on the sensitivity of the instrument and the quality of the chromatographic separation, the optimal mass window will have to be determined by the user. In this example (Figure 5C), the XIC of lysine $(\mathrm{m} / \mathrm{z}=147.1144)$ with a mass window of $10 \mathrm{ppm}$ is shown where lysine in the amino acid standard elutes off the column at 8.03 minutes.

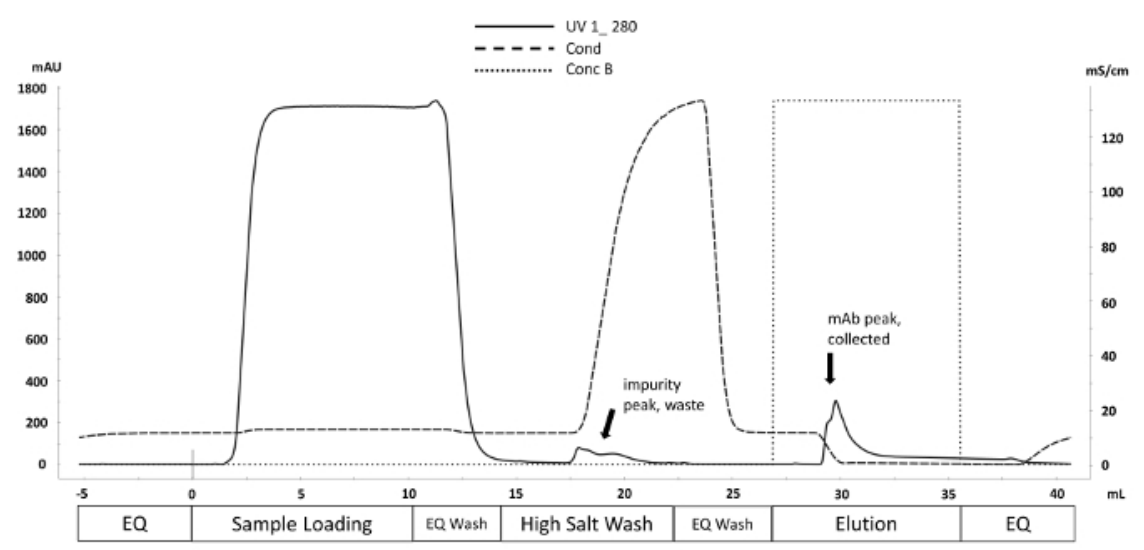

Figure 1. Representative chromatogram of the purification scheme using the Fast Protein Liquid Chromatography (FPLC) technique. Purification method phases corresponding to volume $(\mathrm{mL})$ are labeled along the x-axis. UV absorbance at $280 \mathrm{~nm}$ (mAU y-axis, solid line) is monitored throughout the purification cycle. Non-specifically bound impurities are displaced by increasing conductivity (mS/cm $y$-axis, dashed line) during the High Salt Wash. Antibody is eluted from Protein A column with the introduction of elution buffer (Conc B, dotted line) when the pH decreases to 4 (not shown). Please click here to view a larger version of this figure. 


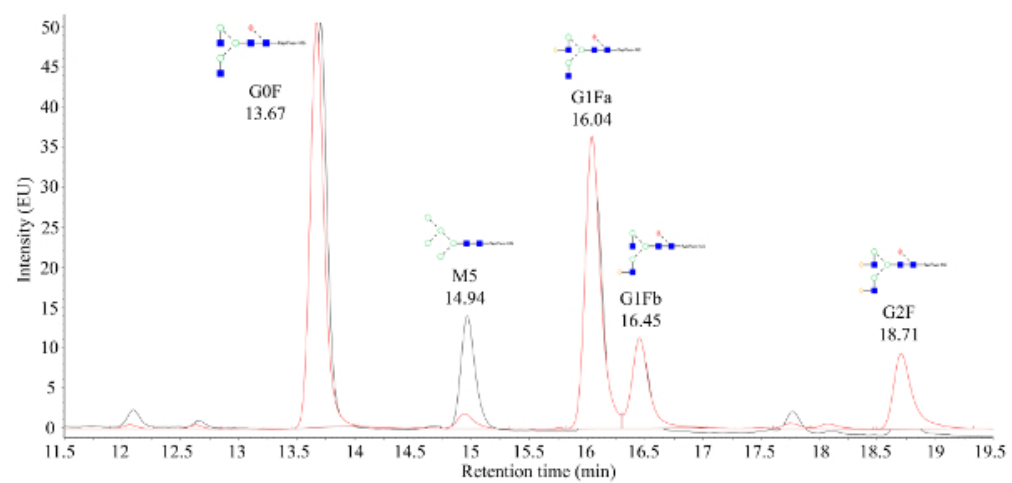

Figure 2. A representative fluorescence chromatogram obtained from tagged glycans that are mass verified. The $x$-axis is retention time (minutes) while the $y$-axis is signal intensity. The peak at 14.94 min represents the Mannose 5 (M5) glycan, where a large difference between the M5 signal strength can be observed between the two samples that are overlaid. Please click here to view a larger version of this figure.

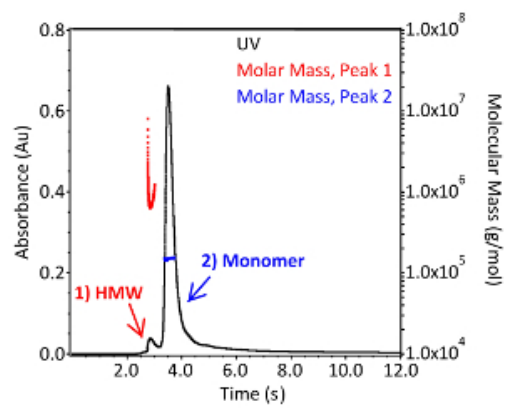

Figure 3. Molecular weight distribution of IgG1 monoclonal antibody. Chromatogram of an intact IgG1 monoclonal antibody separated by size exclusion chromatography in 1x PBS ( $\mathrm{pH} 7.4)$. Absorbance is monitored at $280 \mathrm{~nm}$ (black; left axis) and light scattering and refractive index detectors were used to calculate the absolute molecular weight of each peak (red and blue; right axis). High Molecular Weight species are indicated with the peak labeled "HMW". Please click here to view a larger version of this figure.

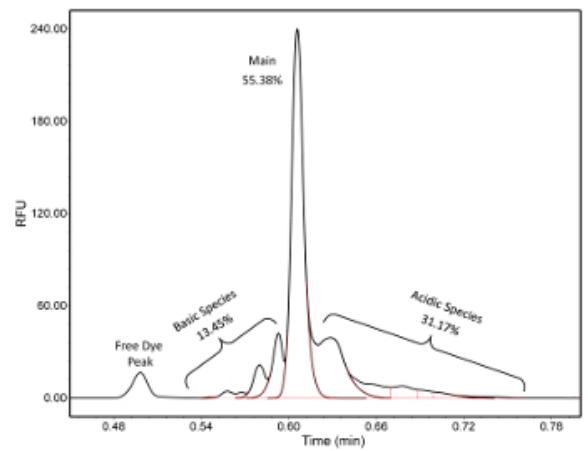

Figure 4. Charge variant profile of a IgG1 monoclonal antibody. This electropherogram is generated on a mCZE platform. A free-dye peak migrates at $\sim 30 \mathrm{~s}$ and is well separated from the IgG1. For quantification, peaks were split into basic, main, and acidic species using instrument data analysis software. The red line outlines the integrated peak areas. Please click here to view a larger version of this figure. 


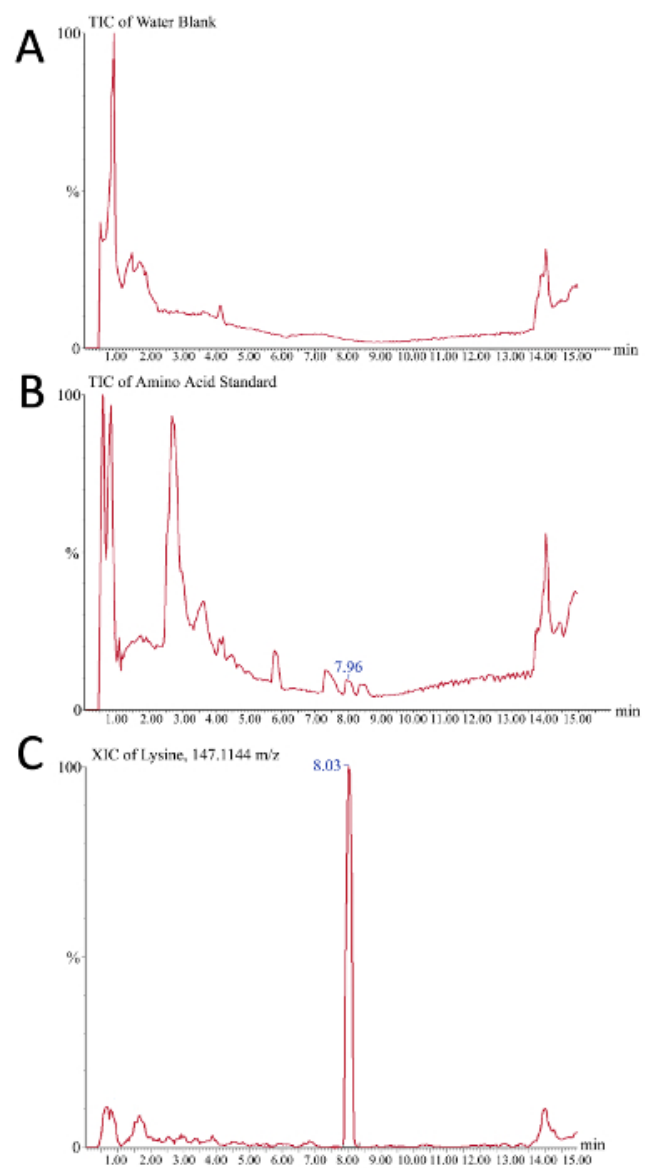

Figure 5. Representative results of the ion chromatograms for mass spectrometry-based amino acid analysis of crude bioreactor media. The $x$-axis is time (minutes) while the $y$-axis is signal intensity (A) A water blank serves as the negative control and reveals the background signal observed over the course of the liquid chromatography gradient (B) The $225 \mathrm{pmol} / \mu \mathrm{L}$ amino acid standard is used here as a positive control, as the individual peaks observed in this total ion chromatogram represent the different amino acids of the standard mix being resolved chromatographically (C) A representative extracted ion chromatogram for $\mathrm{m} / \mathrm{z} 147.1144$, which is lysine. The 7.96 min peak in $B$ corresponds to the 8.03 peak in $\mathrm{C}$ of lysine. Please click here to view a larger version of this figure.

\section{Discussion}

HCCF contains debris and large particles that can clog and destroy costly instrumentation, thus culture clarification is needed before further downstream processing. Centrifugation is generally the first approach to separate cells and other insoluble particles from proteins followed by filtration. This filtered HCCF is then subjected to Fast Protein Liquid Chromatography (FPLC) for purification. Purification of HCCF from automated microbioreactors to obtain the product is an important step in downstream processing. Here, a benchtop FPLC system with a protein A column is used to obtain monoclonal antibodies from the HCCF. Analytics for upstream processes can provide useful insight into cell behavior and guide bioprocess design, helping to obtain a consistent and reliable quality product. Analytics also allows us to link Critical Quality Attributes (CQAs) to upstream and downstream processes. Presented here are four assays that are commonly used in the characterization of monoclonal antibodies. These techniques are robust, reliable, and readily deployable for process and product analysis from a variety of upstream sources which are only partially purified and may still contain residual levels of DNA and HCP.

When cleaning up samples for analytics, an important balance must be struck between creating a sample that is sufficiently clean for analysis while preserving the variability present in the bioreactor. The two most common contaminants impacting product are DNA and HCP, which can be checked by measuring the ratio absorbance at $260 / 280 \mathrm{~nm}$ and through SDS-PAGE or $\mu$ CE-SDS. The assays presented here are not sensitive to low levels of DNA content. The purity of the product is $>95 \%$ pure, as determined by $\mu$ CE-SDS.

Charge variant analysis with a microcapillary electrophoresis system provides a high-throughput method to identify charge variants, with chips and reagents that are relatively easy to implement. The nature of the technique and the chemistry of the labeling reagent are both sensitive to excipients and other primary amines, thus requiring a desalting step for most sample matrixes. From experience, low levels of DNA comigrate with the free-dye from the labeling reaction and do not impact the quality of results. While variability of basic, main, and acidic peak quantification is typically $<1 \%$, higher levels of DNA and other contaminants can increase the variability of the assay. It is extremely important to be consistent with the protein labeling and ensure the prompt use of DMF after removal from the bottle and being mixed with the dye. Lysine and/or histidine standards are recommended as labeling controls. Over time and depending on sample quality, chips can foul or lose the coating on the microfluidics channels, leading to greater noise, the presence of ghost peaks, and greater sample-to-sample variation. To identify this 
occurrence, blanks and a system suitability standard (i.e. NISTmAb) were concurrently analyzed with the samples at regular intervals. When chip issues arise, the chips can be washed with the storage solution or replaced.

The methods used for glycan analysis of therapeutic glycoproteins primarily involves liquid chromatography (LC) and/or mass spectrometry (MS), with lectin microarray analysis gaining popularity as a third option ${ }^{25}$. The method described in this paper uses both LC and MS, which has benefits and disadvantages. Mass spectrometric methods have the advantage of mass verification of the analyzed glycans, which is not possible with LC-based methods using a fluorescent detection output or lectin microarrays. This method uses LC and fluorescence detection to assign glycan identities using retention time comparison to a dextran ladder standard. Fluorescence monitoring allows for increased sensitivity and quantification due to the ease of its detection, where MS alone might not be able to quantify low abundance species due to the poor ionization efficiency of oligosaccharides. The mass information from MS is used to confirm glycan identities, but the processing software does not use mass information as the primary assignment criteria. Hence, without reproducible chromatography and easily resolvable peaks, this method can suffer regarding glycan assignments. Fortunately, the mass information can help with glycan assignments even in situations when the chromatography is subpar, such as shifts in retention time that hinder reproducible glycan assignments. If this method is used without MS, the chromatography must be at the highest level since mass information cannot be used to correct for residence time drift.

The amino acid analysis method described here utilizes LC-MS for rapid quantitation of underivatized amino acids in crude cell culture media. Alternative amino acid analysis methods require amino acid derivatization agents to enable UV detection ${ }^{26}$. The LC-MS method offers important advantages over the LC-UV method: it allows for identification based on both retention time and ion mass as opposed to the LC-UV method, which is limited by a lack of mass characterization. Furthermore, the LC-MS method offers time and reproducibility advantages, as the LC-UV method requires a time-consuming derivatization reaction, which may impart sample variability ${ }^{27}$. However, the injection of crude cell culture media in the LC-MS method can cause detrimental effects on MS signal due to ion skimmer fouling. A calibration ladder is injected frequently as a system suitability check, and sample order is randomized to prevent bias in the data.

The cell culture process for antibody production using microbioreactors is previously described ${ }^{9}$. In this study, detailed protocols for monoclonal antibody characterization methods that maximize data acquired from limited sample volumes are well-defined. Limited amounts of harvested cell culture fluid can sometimes restrict the product information acquired and selection of the right analytical procedures to obtain product quality data is essential. Analytics are important to link together upstream process parameters to the changes in product quality. Here, a guideline is provided for users to characterize mAbs when working with microbioreactors.

\section{Disclosures}

This publication reflects the views of the author and should not be construed to represent FDA's views or policies.

\section{Acknowledgements}

The authors would like to thank Scott Lute for the analytical support he provided. Partial internal funding and support for this work is provided by the CDER Critical Path Program (CA \#1-13). This project is supported in part by an appointment to the Internship/Research Participation

Program at the Office of Biotechnology Products, U.S. Food and Drug Administration, administered by the Oak Ridge Institute for Science and Education through an interagency agreement between the U.S. Department of Energy and FDA.

\section{References}

1. Pharmaceutical cGMPs for the 21st Century: A Risk-Based Approach. (2004).

2. FDA. New Molecular Entity (NME) Drug and New Biologic Approvals., <https://www.fda.gov/Drugs/DevelopmentApprovalProcess/ HowDrugsareDevelopedandApproved/DrugandBiologicApprovalReports/NDAandBLAApprovalReports/ucm373420.htm> (2015).

3. Foltz, I. N., Karow, M., Wasserman, S. M. Evolution and Emergence of Therapeutic Monoclonal Antibodies. Circulation. 127 (22), $2222-2230$ (2013).

4. Kondragunta, B., Drew, J. L., Brorson, K. A., Moreira, A. R., Rao, G. Advances in clone selection using high-throughput bioreactors Biotechnology Progress. 26 (4), 1095-1103 (2010).

5. Hmiel, L., Brorson, K., Boyne, M. Post-translational structural modifications of immunoglobulin $\mathrm{G}$ and their effect on biological activity Analytical \& Bioanalytical Chemistry. 407 (1), 79-94 (2015).

6. Rathore, A. S. Roadmap for implementation of quality by design (QbD) for biotechnology products. Trends in Biotechnology. 27 (9), $546-553$ (2009).

7. International Council for Harmonisation of Technical Requirements for Pharmaceuticals for Human Use. ICH. (ed specifications: test procedures and acceptance criteria for biotechnological/biological products Q6B) (1999).

8. Berkowitz, S. A., Engen, J. R., Mazzeo, J. R., Jones, G. B. Analytical tools for characterizing biopharmaceuticals and the implications for biosimilars. Nature Reviews Drug Discovery. 11 (7), 527-540 (2012).

9. Velugula-Yellela, S. R. et al. Use of high-throughput automated microbioreactor system for production of model IgG1 in CHO cells. Journal of Visualized Experiments. (2018).

10. Largy, E., Cantais, F., Van Vyncht, G., Beck, A., Delobel, A. Orthogonal liquid chromatography-mass spectrometry methods for the comprehensive characterization of therapeutic glycoproteins, from released glycans to intact protein level. Journal of Chromatography $A$. 1498, 128-146 (2017).

11. Yang, J.-M. et al. Investigation of the correlation between charge and glycosylation of IgG1 variants by liquid chromatography-mass spectrometry. Analytical Biochemistry. 448, 82-91 (2014).

12. Agarabi, C. D. et al. Bioreactor Process Parameter Screening Utilizing a Plackett-Burman Design for a Model Monoclonal Antibody. Journal of Pharmaceutical Sciences. 104 (6), 1919-1928 (2015). 
13. Wen, J., Arakawa, T., Philo, J. S. Size-Exclusion Chromatography with On-Line Light-Scattering, Absorbance, and Refractive Index Detectors for Studying Proteins and Their Interactions. Analytical Biochemistry. 240 (2), 155-166 (1996).

14. Veurink, M., Stella, C., Tabatabay, C., Pournaras, C. J., Gurny, R. Association of ranibizumab (Lucentis) or bevacizumab (Avastin) with dexamethasone and triamcinolone acetonide: An in vitro stability assessment. European Journal of Pharmaceutics and Biopharmaceutics. 78 (2), 271-277 (2011).

15. Li, Y., Weiss IV, W. F., Roberts, C. J. Characterization of high-molecular-weight nonnative aggregates and aggregation kinetics by size exclusion chromatography with inline multi-angle laser light scattering. Journal of Pharmaceutical Sciences. 98 (11), $3997-4016$ (2009).

16. Espinosa-de la Garza, C. E. et al. Analysis of recombinant monoclonal antibodies by capillary zone electrophoresis. Electrophoresis. $\mathbf{3 4}$ (8), 1133-1140 (2013).

17. Han, H., Livingston, E., Chen, X. High throughput profiling of charge heterogeneity in antibodies by microchip electrophoresis. Analytical Chemistry. 83 (21), 8184-8191 (2011).

18. Wheeler, T. D. et al.Microchip zone electrophoresis for high-throughput analysis of monoclonal antibody charge variants. Analytical Chemistry. 86 (11), 5416-5424 (2014).

19. Carrillo-Cocom, L. et al.Amino acid consumption in naive and recombinant $\mathrm{CHO}$ cell cultures: producers of a monoclonal antibody. Cytotechnology. 67 (5), 809-820 (2015).

20. Chen, P., Harcum, S. W. Effects of amino acid additions on ammonium stressed CHO cells. Journal of Biotechnology. 117 (3), $277-286$ (2005).

21. Xing, Z. et al. Optimizing amino acid composition of $\mathrm{CHO}$ cell culture media for a fusion protein production. Process Biochemistry. 46 (7) 1423-1429 (2011).

22. Fan, Y. et al. Amino acid and glucose metabolism in fed-batch $\mathrm{CHO}$ cell culture affects antibody production and glycosylation. Biotechnology and Bioengineering. 112 (3), 521-535 (2015).

23. Read, E. K. et al. Fermentanomics informed amino acid supplementation of an antibody producing mammalian cell culture. Biotechnology Progress. 29 (3), 745-753 (2013).

24. Mazzer, A. R., Perraud, X., Halley, J., O'Hara, J., Bracewell, D. G. Protein A chromatography increases monoclonal antibody aggregation rate during subsequent low pH virus inactivation hold. Journal of Chromatography. A. 1415, 83-90 (2015).

25. Zhang, L., Luo, S., Zhang, B. Glycan analysis of therapeutic glycoproteins. MAbs. 8 (2), 205-215 (2016).

26. Wahl, O., Holzgrabe, U. Amino acid analysis for pharmacopoeial purposes. Talanta. 154, 150-163 (2016).

27. Le, A., Ng, A., Kwan, T., Cusmano-Ozog, K., Cowan, T. M. A rapid, sensitive method for quantitative analysis of underivatized amino acids by liquid chromatography-tandem mass spectrometry (LC-MS/MS). Journal of Chromatography B. 944, 166-174 (2014). 